\title{
Application of the Ronchi test to intraocular lenses: A comparison of theoretical and measured results
}

\author{
L. Carretero, C. González, A. Fimia, and I. Pascual
}

\begin{abstract}
We studied the spherical aberration of an intraocular lens, using third-order theory, ray tracing, and bench measurements by the Ronchi test. Good agreement was obtained for these three methods of investigation. In particular we found that the Ronchi test is an accurate method for quantifying this aberration.

Key words: Intraocular lens, Ronchi test, interferometry, optical properties.
\end{abstract}

\section{Introduction}

Analysis of the image quality of any optical system involves studying the aberrations that exist in the system. This analysis is extremely important when dealing with intraocular lenses (IOL's), because they are implanted in cataract patients after their diseased lenses have been removed. In monofocal IOL's the spherical aberration is the most important type of aberration, because these lenses are designed to work with distant objects that are normally on-axis.

Much recent research has been dedicated to the theoretical analysis of the aberrations that are present in IOL's, and studies of both on-axis ${ }^{1,2}$ and off-axis ${ }^{3}$ performance have been done. Off-centering has also been studied from both a theoretical and an experimental approach. 4 Holographic interferometry has been shown to be a useful tool for the analysis of the aberration of wave fronts in IOL's. ${ }^{6}$

In this paper a comparative study of three methods used to study the $A$ coefficient of the spherical aberration of an IOL is carried out. Two of the three methods are theoretical, while the third is experimental and based on application of the Ronchi test. ${ }^{7}$ The Ronchi test is an adequate nondestructive experimen-

The authors are with the Laboratorio de Optica, Departamento Interuniversitario de Optica, Universidad de Alicante, Apartado 99. Alicante E 03080, Spain.

Received 21 October 1992.

0003-6935/93/224132-06\$06.00/0.

(c) 1993 Optical Society of America. tal method, which makes it possible to quantify the image quality of any optical system. This method is particularly appropriate for the study of IOL's because of their size $(\sim 7 \mathrm{~mm}$ in diameter) and their great power (in air: $75 \mathrm{D}$ ). These characteristics make it difficult to use other interferometric systems to measure the aberrations and focal lengths. ${ }^{8}$

In this paper we show that with paraxial theory equations it is also possible to measure the focal length of the IOL with a Ronchi Test in air, thereby obtaining the value of that focal length when it is immersed in vitreous humor.

\section{Comparison of Third-Order Theory and Exact Ray Tracing}

The wave-front aberration of an optical system in the exit pupil can be written as?

$$
\begin{aligned}
W= & A\left(x^{2}+y^{2}\right)^{2}+B y\left(x^{2}+y^{2}\right) \\
& +C\left(x^{2}+3 y^{2}\right)+D\left(x^{2}+y^{2}\right),
\end{aligned}
$$

where $A, B, C$, and $D$ are the spherical aberration, coma, astigmatism, and defocusing coefficients, respectively, and $x, y$ are the coordinates of the exit pupil of the optical system. From an optical-geometric point of view, if we consider third-order theory and keep in mind that an IOL is thin, the spherical aberration of this kind of lens can be determined with the following equation ${ }^{9}$.

$$
S=\frac{h^{2}}{f}\left(\alpha q^{2}+\beta p q+\gamma p^{2}+\zeta\right)
$$


where

$$
\begin{aligned}
\alpha=\frac{n^{2}(\mu+2 n)}{8 \mu(\mu-n)^{2}}, & \beta=\frac{n(\mu+n)}{2 \mu(\mu-n)}, \\
\gamma=\frac{3 \mu+2 n}{8 \mu}, & \zeta=\frac{\mu^{2}}{8(\mu-n)^{2}}, \\
q=\frac{r_{2}+r_{1}}{r_{2}-r^{1}}, & p=\frac{z_{i}{ }^{\prime}+z_{0}}{z_{i}{ }^{\prime}-z_{0}}
\end{aligned}
$$

Parameter $h$ is the height above the optical axis where the light hits the lens, $f$ is the focal length of the lens, and $p$ and $q$ are the position parameter and the bending factor, respectively, $r_{1}$ and $r_{2}$ are the curvature radii of the first and second surfaces of the lens, the object and image distances are denoted by $z_{0}$ and $z_{i}{ }^{\prime}, \mu$ is the refractive index of the lens, and $n$ is the index of the medium that surrounds the lens. To reproduce the experimental conditions that we will subsequently use for the Ronchi test, we assumed that the lens is surrounded by air, thereby making $n=1$.

Given that the $A$ coefficient of the spherical aberration was measured experimentally, we must keep in mind that this coefficient is related to $S$ (the thirdorder spherical aberration) as shown by the following equation ${ }^{7}$ :

$$
A=\frac{S}{4 f^{2} h^{2}} .
$$

We can use third-order theory to deduce that the $A$ coefficient depends on the geometric parameters of the lens according to

$$
\begin{aligned}
A= & \frac{1}{32 f^{3}}\left[\frac{\mu+2}{\mu(\mu-1)^{2}} q^{2}+\frac{4(\mu+1)}{\mu(\mu-1)} p q\right. \\
& \left.+\frac{3 \mu+2}{\mu} p^{2}+\frac{\mu^{2}}{(\mu-1)^{2}}\right] .
\end{aligned}
$$

This equation was applied to an IOL that has one flat and one convex surface. This means that, depending on the side of the lens that the light hits, we can assume that the lens is either plano-convex or convexo-plane, that is, that the bending factor is -1 or 1 , respectively.

Given that the lens was made in poly(methyl methacrylate), we used the refraction index that corresponded to that material for a wavelength of 633 $\mathrm{nm}$ with an $\mathrm{He}-\mathrm{Ne}$ laser, $\mu=1.490$, so that we could reproduce the experimental conditions for the Ronchi test and compare the results.

For this same reason the position parameter that we used was $p=-1$; in other words the object was at infinity.

To calculate the $A$ coefficient, both theoretically and experimentally, we obtained the value of the focal length of the IOL with the Ronchi test. To do so the diffraction grating was placed next to the IOL (see

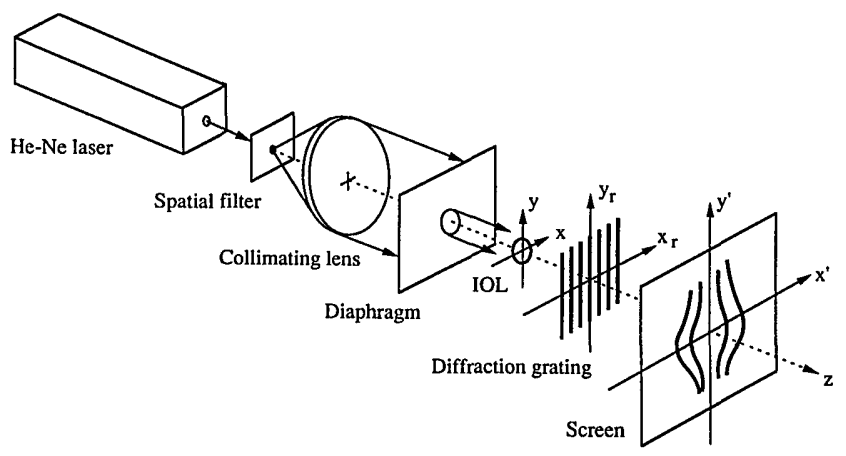

Fig. 1. Experimental setup: $x, y$, coordinates in the exit pupil plane; $x_{r}, y_{r}$, coordinates in the grating plane; $x^{\prime}, y^{\prime}$, coordinates in the image plane; $z$, optical axis.

Fig. 1) and moved with a micrometric screw (with a sensitivity of $10 \mu \mathrm{m}$ ) until an interference figure appeared on the screen that was fusiform in shape. For the plano-convex orientation the distance of the lens from the grating was the focal length of the IOL. The experimental value of the focal length of the lens obtained was

$$
f=13.15 \pm 0.02 \mathrm{~mm} \text {. }
$$

To calculate the power of the IOL immersed in vitreous humor, paraxial optics formulas are used:

$$
P_{h v}=\frac{\mu-n_{h v}}{(\mu-1)} P_{\text {air }}
$$

where $P_{h v}$ is the power of the lens in vitreous humor, $P_{\text {air }}$ is the power of the lens in air, and $n_{h v}$ is the refraction index of vitreous humor. This relationship, together with the coefficient of the spherical aberration, permits us to evaluate the contribution of the IOL to the overall spheric aberration of the eye-lens system and thereby estimate the influence that the IOL has on the quality of the final image.

When these data are substituted in the expression for the $A$ coefficient, the following values were obtained in air:

for plano-convex orientation

$$
A=(5.08 \pm 0.02) \times 10^{-4} \mathrm{~mm}^{-3},
$$

for convexo-plane orientation

$$
A=(1.333 \pm 0.006)-10^{-4} \mathrm{~mm}^{-3} .
$$

A graphic tracing of the rays confirmed our results above, and the Ronchi test was used to measure the $A$ coefficient experimentally.

Through graphic tracing we found that the image position for an object in infinity given a certain height $h$ is the following:

plano-convex orientation:

$$
s_{h}^{\prime}=h\left\{\frac{1}{\tan \left[\sin ^{-1}\left(\frac{n h}{r}\right)-\sin ^{-1}\left(\frac{h}{r}\right)\right]}\right\}-a ;
$$


convexo-plane orientation:

$$
x_{h}{ }^{\prime}=\frac{(a-e) \tan \beta+h}{\tan \left[\sin ^{-1}(n \sin \beta)\right]} .
$$

Both distances were measured in relation to the last surface of the lens (see Fig. 2) with $h$ being the height of incidence on the lens that varied from zero (paraxial focal length) to the semidiameter of the lens, when the diameter is $6.96 \pm 0.05 \mathrm{~mm}, e$ is the thickness of the lens and has a value of $1.32 \pm 0.01$ $\mathrm{mm}, n$ is the refraction index, and $r$ is the curvature radius. Parameters $\alpha$ and $\beta$ can be found by using

$$
\begin{aligned}
& a=r-\left(r^{2}-h^{2}\right)^{1 / 2}, \\
& \beta=\sin ^{-1} \frac{h}{r}-\sin ^{-1} \frac{h}{n r} .
\end{aligned}
$$

The value of the $A$ coefficient with this method is obtained from adjustment by the least squares of the line obtained by representing $\left(S / 4 f^{2}\right)$ as a function of $h^{2}$ (see Fig. 3) where the value of $S$ is obtained through ray tracing by subtracting all the image positions for each height from the paraxial focal point.

Both the calculation of $S$ and the numerical adjustment were done on a personal computer using a program designed for this purpose. The values obtained in air were

plano-convex orientation:

$$
A=(5.63 \pm 0.03) \times 10^{-4} \mathrm{~mm}^{-3},
$$

convexo-plane orientation:

$$
A=(1.4 \pm 0.1) \times 10^{-4} \mathrm{~mm}^{-3},
$$

with the respective regression coefficients being 0.9991 and 0.9997 .
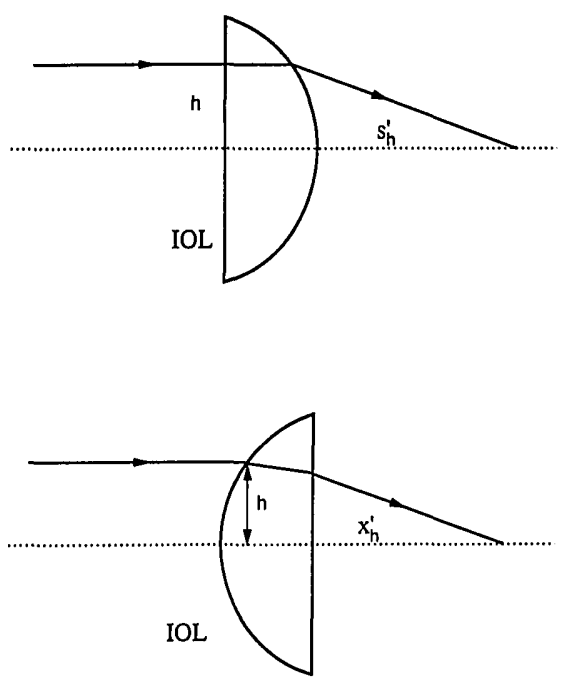

Fig. 2. Ray-tracing scheme for (a) a plano-convex orientation and (b) a convexo-plane orientation; $h$, ray height; $S_{h}{ }^{\prime}$, back focal segment for plano-convex orientation; $X_{h}{ }^{\prime}$, back focal segment for convex-plane orientation.
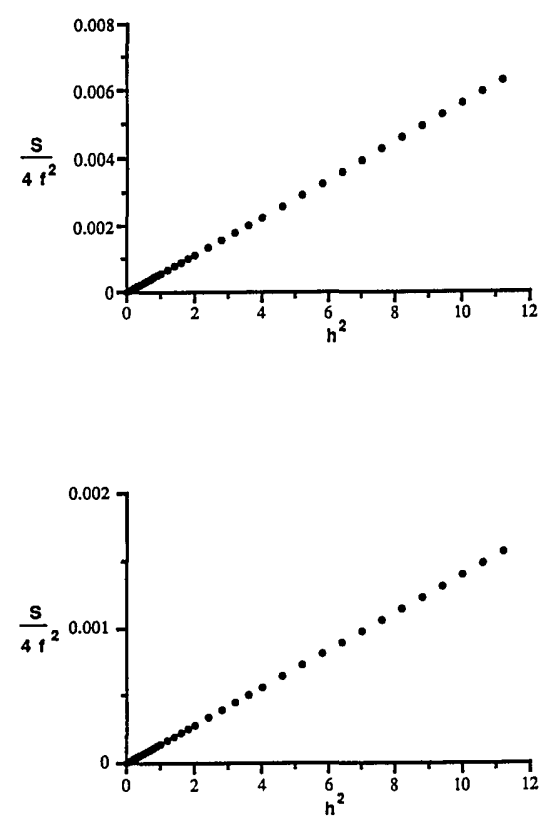

(b)

Fig. 3. Function of incident height $\left(\mathrm{S} / 4 f^{2}\right)$ for (a) plano-convex orientation and (b) convexo-plane orientation; $h^{2}$, ray height.

\section{Optical Bench Measurements: Ronchi Test}

The Ronchi test was used to measure the $A$ coefficient of spherical aberration and the focal length of IOL's. ${ }^{7}$ The experiment setup is shown in Fig. 1, where the $\mathrm{He}-\mathrm{Ne}$ laser has $10 \mathrm{~mW}$ of power and the collimating lens has a focal length of $300 \mathrm{~mm}$ (white light) and a diameter of $10 \mathrm{~mm}$; that is, the aperture of the collimating lens is 0.03 . This ensures that the collimating lens does not produce any aberrations because the aperture is small compared with the aperture of the IOL we studied, which was 0.5 . The diffraction grating frequency was 330 lines $/ \mathrm{mm}$.

This test consists of situating a diffraction grating with the appropriate frequency near the image point of an optical system. The light emerging from the diffraction grating produces the different diffracted orders, which interfere with one another and result in real nonlocalized interference fringes that can be seen on a screen (see Fig. 1).

When diffraction theory is applied, ${ }^{10}$ the intensity distribution on the exit pupil is given by

$$
\begin{aligned}
I(x, y)= & \left|B_{0}\right|^{2}+\left|B_{1}\right|^{2}+2 B_{0} B_{1} \\
& \times \cos \left\{\frac{2 \pi}{\lambda}\left[W(x, y)-W\left(x+\frac{c \lambda}{d}, y\right)\right]\right\},
\end{aligned}
$$

where we have supposed that the grating has an appropriate period $d$ to produce interference patterns of no more than zero-order and first-order diffraction. $B_{0}$ and $B_{1}$ are the Fourier coefficients of the gratings, $x$ and $y$ are the coordinates of the exit pupil, $d$ is the grating period, $c$ is the radius of curvature of the wave front, and $\lambda$ is the wavelength of the incident beam.

It can be seen from Eq. (10) that bright fringes 
appear whenever

$$
w(x, y)-w\left(x+\frac{c \lambda}{d}, y\right)=-m \lambda
$$

If we now take the center point between the two shears of the wave fronts as a new point organ, we find that

$$
w\left(x+\frac{c \lambda}{2 d}, y\right)-w\left(x-\frac{c \lambda}{2 d}, y\right)=-m \lambda .
$$

Fixing the $y$ variable, we can use

$$
\frac{\partial w}{\partial x} \frac{c \lambda}{d}=w\left(x+\frac{c \lambda}{2 d}, y\right)-w\left(x-\frac{c \lambda}{2 d}, y\right)
$$

and then we find that the fringes of the interferogram are related to the aberration function of wave $W(x, y)$ according to

$$
\frac{\partial W}{\partial x}=-\frac{m d}{c},
$$

where $m$ is the order of interference.

For the interference fringes to be seen clearly, the frequency $v$ of the grating must allow only consecutive diffracted orders to interfere, as we have mentioned, and the conditions that bring this about are

$$
\frac{\phi}{\lambda}\left\{\frac{1}{\left[c^{2}+\left(\frac{\phi}{2}\right)^{2}\right]^{1 / 2}}\right\}>v>\frac{\phi}{2 \lambda}\left\{\frac{1}{\left[c^{2}+\left(\frac{\phi}{2}\right)^{2}\right]^{1 / 2}}\right\},
$$

where $\phi$ is the diameter of the lens, $\lambda$ is the wavelength, and $c$ is the distance from the lens to the diffraction grating.

Considering that when one is working on-axis the wave aberration can be only spherical, the experimental interferogram (see Fig. 4) is represented by Eqs.

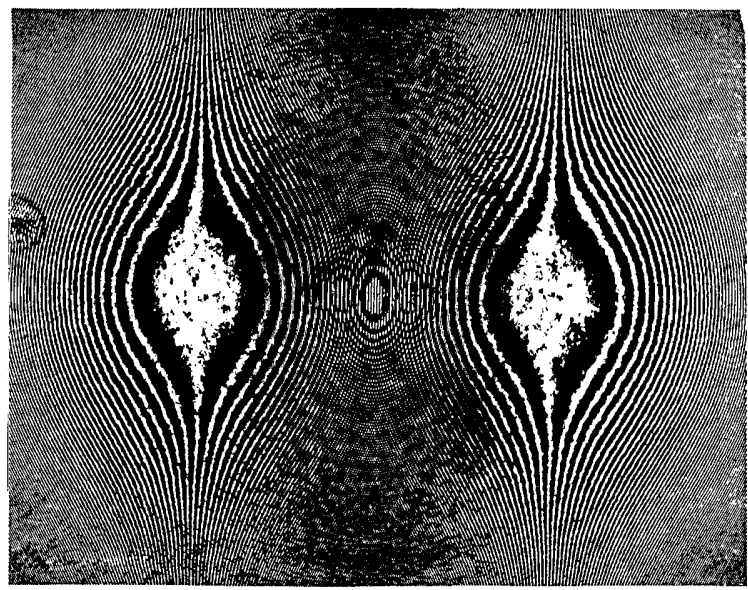

Fig. 4. Experimental interferogram obtained with a planoconvex lens.
(1) and (14) by

$$
4 A x\left(x^{2}+y^{2}\right)=-\frac{m d}{c} .
$$

The value of the interfringe distance is $d=$ $0.002996 \pm 0.000005 \mathrm{~mm}, c=13.15 \pm 0.02 \mathrm{~mm}$ for the plano-convex orientation, and $c=12.82 \pm 0.02$ $\mathrm{mm}$ for the convexo-plane orientation. A consequence of the interference of the zero order with the first order diffracted by the diffraction grating is an interferential pattern appearing on the observation screen at $650 \mathrm{~mm}$ from the grating. This is recorded on a photographic plate because interferential phenomena are extremely sensitive to external perturbations. Once the plate is developed, we place a millimetered transparent sheet on it that permits us to measure the $x^{\prime}$ and $y^{\prime}$ coordinates of each interference fringe, which is at the same time characterized by the diffraction order. Afterward the $x^{\prime}$ and $y^{\prime}$ coordinates are transferred to the exit pupil plane $x-y$. For the experimental setup we deduce the proportionality relationship between $\left(x^{\prime}, y^{\prime}\right)$ and $(x, y)$ :

$$
x=\frac{x^{\prime} c}{D^{\prime}} \quad y=\frac{y^{\prime} c}{D^{\prime}},
$$

where $D^{\prime}$ is the distance from the grating to the screen. To obtain the $A$ coefficient of the spherical

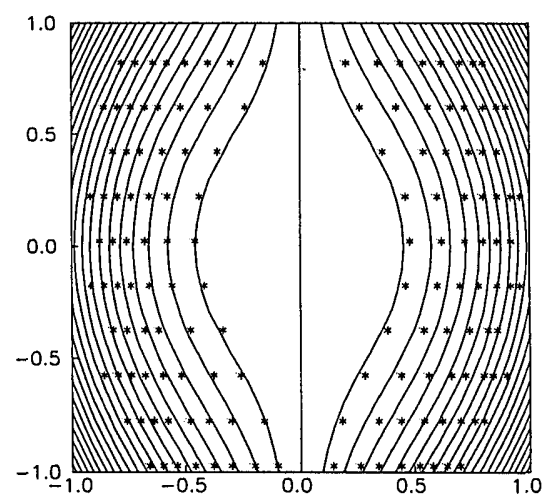

(a)

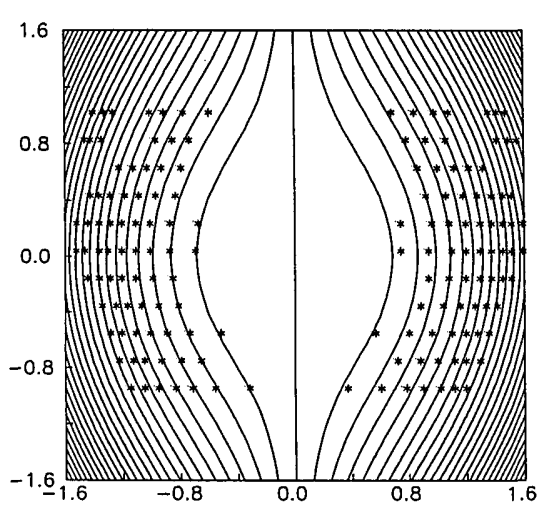

Fig. 5. Theoretical interferogram (continuous line) and experimental digitalized interferogram (*) for (a) plano-convex orientation and (b) convexo-plane orientation. Curves indicate $\partial w / \partial x=$ constant. 
aberration, a least-squares adjustment method was used and the theoretical surface (see Fig. 6) was adjusted to the experimental surface formed by coordinate points $\left(x_{i}, y_{i}, m_{i} d / c\right)$ obtained from the interferogram (see Fig. 5). To do this, we define the function

$$
T=\sum_{i=1}^{N}\left[\frac{m_{i} d}{c}-4 A x_{i}\left(x_{i}{ }^{2}+y_{i}^{2}\right)\right]^{2}
$$

by using the minimum condition

$$
\frac{\partial T}{\partial A}=0
$$

We deduce that

$$
A=\frac{\sum_{i=1}^{N} \frac{m_{i} d}{c} x_{i}\left(x_{i}^{2}+y_{i}^{2}\right)}{4 \sum_{i=1}^{N} x_{i}^{2}\left(x_{i}^{2}+y_{i}^{2}\right)^{2}}
$$
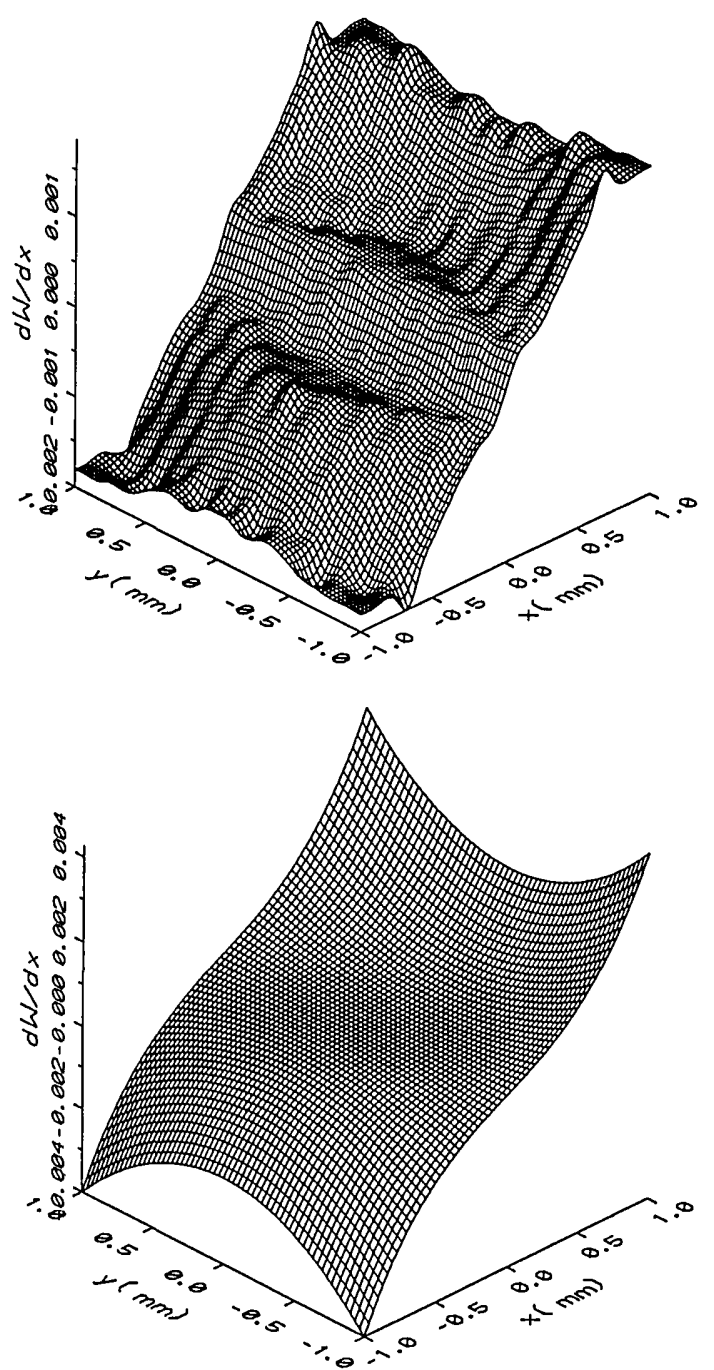

Fig. 6. Function of exit pupil coordinates $(\mathrm{d} W / \mathrm{d} X)$ : (a) experimental surface; (b) theoretical surface.
To obtain the $A$ coefficient, a program specially designed for this purpose was used, and the results we obtained in air are as follows:

plano-convex orientation:

$$
A=(5.16 \pm 0.07) \times 10^{-4} \mathrm{~mm}^{-3},
$$

convexo-plane orientation:

$$
A=(1.54 \pm 0.03) \times 10^{-4} \mathrm{~mm}^{-3},
$$

where the regression coefficients were 0.997 and 0.997 , respectively.

Regarding Fig. 6, the differences between the two representations are shown by experimental [Figs. 6(a)] and theoretical [Fig. 6(b)] results. The theoretical representation is a continuous function, while the experimental one was obtained by using discontinuous points determined experimentally by the Ronchi test. Therefore the discrepancy between the two is due mainly to our experimental errors. We must also keep in mind that there are errors in curvature (lack of constancy in the bending factor) in the manufacturing process of the IOL surfaces and that these affect the experimental interferogram that we obtained. However, we do not know the magnitude of these errors in curvature, even though we do know that they exist. If a more complex and complete measurement system were used, for example, a digital image analysis system, the experimental errors could be reduced, and the differences between the theoretical and experimental representations would be almost exclusively a result of the errors in curvature on the surfaces of the lens, since it is virtually impossible to make perfectly spherical lenses.

\section{Conclusions}

The Ronchi test, third-order theory, and a ray-tracing computer program were used to study the spherical aberration in the IOL's. Taking into account that third-order theory is an approximation and that there is a $1 \%$ margin of error in experimental results when the Ronchi test is used, the results we obtained with each method match quite well, especially if we take into account that the theoretical models are simplified, in other words, they are approximations. Nevertheless their results are comparable with the experimental results, which are rigorously accurate. We should also keep in mind that the sources of error for each of the three methods are different.

At the same time the results from the three methods used in this paper show the influence of the bending factor on the quality of the image. One thing that can be deduced from Eq. (4) and is clearly shown in Refs. 11 and 12 is that the $A$ coefficient of the spherical aberration depends on its position parameter and its bending factor. For the position parameter used we can see that the convexo-plane orientation is the one that produces the best image quality by obtaining the lowest value for coefficient $A$. For other position parameters the bending factor that 
produces the best image quality might be different. In addition to the influence of the IOL bending factor on spherical aberrations, its influence on the final image quality that could be achieved for a cataract patient with an implanted IOL could also be analyzed. ${ }^{3}$

These results also show that the Ronchi test is an excellent method for the nondestructive analysis of IOL's, which, because of their size and high power, create quite restrictive experimental conditions.

The analysis that was carried out here can be continued by calculating off-axis wave aberrations, astigmatism, and coma, thereby the total aberration of the wave for each type of lens is obtained. These results may be used to describe the optical properties of lenses, or they may be used to monitor manufacturing quality, since each primary aberration has an interference figure that is characteristically different and that may be recognized from the shape of the fringes. The higher the number of aberrations a system has, the more complex its interferogram will be. ${ }^{11}$ The method could also be used to develop other IOL designs and to test their effect on off-axis performance.

\section{References}

1. D. A. Atchison, "Optical design of intraocular lenses. I. Onaxis performance," Optom. Vis. Sci. 66, 492-506 (1989).

2. D. A. Atchison, "'Optical design of intraocular lenses. III.
On-axis performance in the presence of lens displacement," Optom. Vis. Sci. 66, 671-681 (1989).

3. D.A. Atchison, "Optical design of intraocular lenses. II. Offaxis performance," Optom. Vis. Sci. 66, 579-590 (1989).

4. D. A. Atchison, "Refractive errors induced by displacement of intraocular lenses within the pseudoaphakic eye," Optom. Vis. Sci. 66, 146-152 (1989).

5. J. D. Auran, C. J. Koester, and A. Donn, "In vivo measurement of posterior chamber intraocular decentration and tilt," Arch. Ophthalmol. 108, 75-79 (1990).

6. L. Carretero, R. Fuentes, and A. Fimia, "Measurement of wave aberrations of intraocular lenses through holographic interferometry," in Holographic Optics, III: Principles and Applications, G. M. Morris, ed., Proc. Soc. Photo-Opt. Instrum. Eng. 1507, 458-462 (1991).

7. D. Malacara, Optical Shop Testing (Wiley, New York, 1978).

8. L. Carretero, R. Fuentes, and A. Fimia, "Measurement of spherical aberration of intraocular lenses with the Ronchi test," Optom. Vis. Sci. 69, 190-192 (1992).

9. W. T. Welford, Aberration of Optical System (Hilger, Boston, Mass., 1986), Chap. 8.

10. R. Barakat, "General diffraction theory of optical aberration tests, from the point of view of spatial filtering," J. Opt. Soc. Am. 59, 1432-1439 (1969).

11. V. N. Mahajan, Aberration Theory Made Simple (Society of Photo-Optical Instrumentation Engineers, Bellingham, Wash., 1991), Vol. TT06, Chap. 12.

12. F. A. Jenkins and H. E. White, Fundamentals of Optics (McGraw-Hill, New York, 1976), Chap. 9.

13. A. Fimia, J. L. Alió, I. Pascual, and A. Beléndez, "New theoretical matrix formula for intraocular lens calculation using the optimal bending factor," J. Cataract Refr. Surg. 19, 293-297 (1993). 